\title{
Treatment of the Coulomb interaction in momentum space calculations of proton elastic scattering
}

\author{
R. Crespo* and J. A. Tostevin \\ Department of Physics, University of Surrey, Guildford, Surrey GU2 5XH, United Kingdom
}

(Received 13 November 1989)

\begin{abstract}
A practical, numerically stable procedure for a more accurate treatment of the Coulomb interaction in momentum space calculations of proton elastic scattering is presented. The accuracy of the method is compared with other prescriptions in use in the literature and is shown to produce accurate calculations of scattering observables.
\end{abstract}

\section{INTRODUCTION}

It is now well established ${ }^{1,2}$ that the nonlocality of the first order Kerman-McManus-Thaler (KMT) optical potential $^{3}$ for nucleon-nucleus scattering must be treated accurately in order to reproduce experimental data for proton-nucleus elastic scattering at intermediate energies. It thus proves very convenient to perform the associated numerical calculations in momentum space.

It has been shown clearly, in the recent calculations of Elster et al., ${ }^{2}$ that intermediate energy calculations of nucleon-nucleus scattering are highly sensitive to the details of the theoretical input to the calculations, a sensitivity which reveals itself most often near interference minima in spin-dependent observables and at larger scattering angles. The effects of the inclusion of the Coulomb interaction are, however, manifest in the same angular regions. Thus, to be able to probe details of our description of the strong nucleon-nucleus interaction, by comparison with the now high precision experimental data, it is vital that the effects of the Coulomb interaction can be included accurately to as large a scattering angle as can reasonably be achieved.

The common approach to incorporating the Coulomb potential in momentum space, and that adopted here, is to use, in the solution of the integral equation, a free particle Green's function and to add the momentum space representation of the Coulomb interaction to the finite ranged (in configuration space) nuclear interactions $V_{N}$. This procedure is, however, complicated by the $1 / q^{2}$ singularity in the momentum space representation of the Coulomb interaction. Approximate prescriptions, to overcome this difficulty, have been discussed in the literature $\mathrm{t}^{4-6}$ and are examined here. We present an alternative approximate, simple and stable method which yields more accurate calculations of reaction observables.

\section{THE ELASTIC SCATTERING AMPLITUDE}

In the scattering of a spin- $\frac{1}{2}$ charged particle from a spinless target, the scattering amplitude can be written

$$
f(\theta)=A(\theta)+i \sigma \cdot \hat{n} C(\theta),
$$

where $A$ and $C$ are the central and spin-dependent Wolfenstein amplitudes

$$
\begin{aligned}
& A(\theta)= f_{C}^{p t}(\theta) \\
&+\frac{1}{k} \sum_{L=0}^{\infty} \exp \left(2 i \sigma_{L}\right)\left[(L+1) T_{L+}(N)\right. \\
&\left.+L T_{L-}(N)\right] P_{L}(\cos \theta),
\end{aligned}
$$

and

$$
C(\theta)=\frac{1}{k} \sum_{L=1}^{\infty} \exp \left(2 i \sigma_{L}\right)\left[T_{L+}(N)-T_{L-}(N)\right] P_{L}^{1}(\cos \theta),
$$

and $\hat{n}$ is the unit vector normal to the scattering plane, $\hat{n}=\mathbf{k} \times \mathbf{k}^{\prime} /\left|\mathbf{k} \times \mathbf{k}^{\prime}\right|$. In Eq. $(2), f_{C}^{p t}(\theta)$ is the Coulomb scattering amplitude due to a point charge $(Z e)$ and $k$ is the asymptotic wave number of the projectile in the nucleon-nucleus $(N-A)$ center of mass frame. The $T_{L \pm}(N)$ are the Coulomb-modified nuclear partial wave transition amplitudes, where $L \pm$ denotes the orbital and total angular momenta, $J=L \pm \frac{1}{2}$. The $T_{L \pm}(N)$ measure the deviations from point Coulomb scattering due to short ranged nuclear interactions, $V_{N}$, and from the Coulomb interaction, $V_{C}$, of the target of charge density $\rho(R)$. They are defined

$$
T_{L \pm}(N)=\frac{\left\{\exp \left[2 i \delta_{L \pm}(N)\right]-1\right\}}{2 i}
$$

where the $\delta_{L \pm}(N)$ are the Coulomb-modified nuclear phase shifts, defined in configuration space through the asymptotic form of the radial wave functions

$$
\psi_{L \pm}(R) \rightarrow F_{L}(k R)+T_{L \pm}(N) H_{L}^{+}(k R),
$$

where $H_{L}^{+}=G_{L}+i F_{L}, F_{L}$ and $G_{L}$ are the regular and irregular Coulomb functions, and $\psi_{L \pm}$ is the solution of the radial Schrödinger equation for potential $V_{N}+V_{C}$. We now briefly review procedures, discussed in the literature, for the approximate evaluation of the $T_{L \pm}(N)$ in the presence of the Coulomb interaction.

\section{APPROXIMATE METHODS}

In the crudest approximation, when calculating the $T_{L \pm}(N)$ in momentum space, it has been supposed that 
the dominant effects of the Coulomb interaction are contained within the Coulomb-nuclear interference, introduced in Eqs. (1)-(3), and in the Coulomb phase factor $\exp \left(2 i \sigma_{L}\right)$ entering in Eqs. (2) and (3). Thus it has been suggested that one could evaluate ${ }^{4}$

$$
T_{L \pm}^{(\mathrm{I})}(N) \approx T_{L \pm}\left(V_{N}\right),
$$

where $T_{L \pm}\left(V_{N}\right)$ is to be calculated in the complete absence of the Coulomb interaction. That is $T_{L \pm}^{(\mathrm{I})}(N)$, in configuration space, is defined by the equation

$$
\psi_{L \pm}^{(\mathrm{I})}(R) \rightarrow \hat{j}_{L}(k R)+T_{L \pm}^{(\mathrm{I})}(N) \hat{h}_{L}^{+}(k R),
$$

where $\hat{h}_{L}^{+}=\hat{n}_{L}+i \hat{j}_{L}, \hat{j}_{L}$ and $\hat{n}_{L}$ are the Riccati-Bessel and Neumann functions, $\hat{j}_{L}(x)=x j_{L}(x)$, etc., and $\psi_{L \pm}^{(\mathrm{I})}$ is the solution of the radial equation in the presence of the nuclear interaction $V_{N}$ alone. We call this method (I).

An attempt to include, to some extent, the Coulomb interaction effects in the calculation of the $T_{L \pm}(N)$ has been used by Picklesimer et al. ${ }^{5}$ They make the replacement

$$
T_{L \pm}^{(\mathrm{II})}(N) \approx T_{L \pm}\left(V_{N}+V_{C}-V_{C}^{p t}\right)
$$

where $V_{C}^{p t}$ is the Coulomb interaction due to a point source of charge $Z e$. As the notation implies, $T_{L \pm}\left(V_{N}+V_{C}-V_{C}^{p t}\right)$ is the partial wave transition amplitude, defined by Eq. (7), with $\psi_{L \pm}^{(\text {II })}$ calculated from the entirely short ranged interaction $V_{N}+V_{C}-V_{C}^{p t}$.

A more sophisticated technique, method (III), for the evaluation of the $T_{L \pm}(N)$ was proposed by Vincent and Phatak. ${ }^{6}$ The method is in principle exact, provided there exists a cut-off radius, $R_{\text {cut }}$, beyond which $V_{N}$ can be assumed to have vanished and $V_{C}$ to have its asymptotic form, $V_{C}(R)=Z e^{2} / R$. The technique is to calculate, in momentum space, an intermediate set of partial wave amplitudes $T_{L \pm}\left(V_{N}+V_{C ; R_{\text {cut }}}\right)$ using the momentum space form,

$$
\begin{aligned}
& V_{C ; R_{\text {cut }}}(q)=\left\langle\mathbf{k}^{\prime}\left|V_{C ; R_{\text {cut }}}\right| \mathbf{k}\right\rangle \\
& =\frac{Z e^{2}}{2 \pi^{2} q^{2}}\left[\rho(q)-\cos \left(q R_{\mathrm{cut}}\right)\right], \\
& \rho(0)=1,
\end{aligned}
$$

where $q=\left|\mathbf{k}^{\prime}-\mathbf{k}\right|$. The potential (9) corresponds to the finite ranged potential $V_{C ; R_{\text {cut }}}$, where

$$
\begin{aligned}
& V_{C ; R_{\text {cut }}}(R)=V_{C}(R) ; \quad R \leq R_{\text {cut }}, \\
& V_{C ; R_{\text {cut }}}(R)=0 ; \quad R>R_{\text {cut }} .
\end{aligned}
$$

The requirement that this cut-off solution should match smoothly with the exact solution, Eq. (5), at the cut-off radius $R_{\text {cut }}$, i.e.,

$$
\begin{aligned}
\psi_{L \pm}^{(\mathrm{III})}\left(R_{\text {cut }}\right)= & \hat{j}_{L}\left(k R_{\text {cut }}\right) \\
& +T_{L \pm}\left(V_{N}+V_{C ; R_{\text {cut }}}\right) \hat{h}_{L}^{+}\left(k R_{\text {cut }}\right) \\
\propto & F_{L}\left(k R_{\text {cut }}\right)+T_{L \pm}^{(\mathrm{III})}(N) H_{L}^{+}\left(k R_{\text {cut }}\right),
\end{aligned}
$$

determines the transition amplitudes $T_{L \pm}^{(\mathrm{III})}(N)$ according to

$$
T_{L \pm}^{(\mathrm{III})}(N)=\frac{T_{L \pm}\left(V_{N}+V_{C ; R_{\mathrm{cut}}}\right)\left[F_{L}, \hat{h}_{L}\right]+\left[F_{L}, \hat{j}_{L}\right]}{\left[\hat{j}_{L}, H_{L}\right]+T_{L \pm}\left(V_{N}+V_{C ; R_{\text {cut }}}\right)\left[\hat{h}_{L}, H_{L}\right]},
$$

where the square brackets denote Wronskians, evaluated at the cut radius, e.g.,

$$
\left[F_{L}, \hat{j}_{L}\right]=\left[\hat{j}_{L} \frac{d F_{L}}{d R}-F_{L} \frac{d \hat{j}_{L}}{d R}\right]_{R_{\mathrm{cut}}}
$$

\section{THE SUBTRACTED MOMENTUM SPACE METHOD}

In this section we propose an alternative approximate method, method (IV), for treating the Coulomb interaction in momentum space. Like Vincent and Phatak, we proceed from calculations of the partial wave transition amplitudes $T_{L \pm}\left(V_{N}+V_{C ; R_{\text {cut }}}\right)$, calculated in the presence of the cut-off Coulomb interaction.

These transition amplitudes can be written

$$
\begin{aligned}
T_{L \pm}\left(V_{N}+V_{C ; R_{\text {cut }}}\right)= & \exp \left[2 i \delta_{L \pm}\left(V_{C ; R_{\text {cut }}}^{p t}\right)\right] T_{L \pm}^{(\text {IV })}(N) \\
& +T_{L \pm}\left(V_{C ; R_{\text {cut }}}^{p t}\right)
\end{aligned}
$$

where the $T_{L \pm}\left(V_{C ; R_{\text {cut }}}^{p t}\right)$ describe scattering from a cut-off point Coulomb interaction,

$$
V_{C ; R_{\text {cut }}}^{p t}(q)=\frac{Z e^{2}}{2 \pi^{2} q^{2}}\left[1-\cos \left(q R_{\text {cut }}\right)\right],
$$

and where

$$
T_{L \pm}\left(V_{C ; R_{\mathrm{cut}}}^{p t}\right)=\frac{\left\{\exp \left[2 i \delta_{L \pm}\left(V_{C ; R_{\mathrm{cut}}}^{p t}\right)\right]-1\right\}}{2 i},
$$

with $\delta_{L \pm}\left(V_{C ; R_{\text {cut }}}^{p t}\right)$ the associated phase shifts. Our approximation to the exact amplitude $T_{L \pm}(N)$ is

$$
T_{L \pm}^{(\mathrm{IV})}(N)=\frac{\left\{\exp \left[2 i \bar{\delta}_{L \pm}(N)\right]-1\right\}}{2 i}
$$

where the phase shift $\bar{\delta}_{L \pm}(N)$ is defined by the relation

$$
\bar{\delta}_{L \pm}(N)=\delta_{L \pm}\left(V_{N}+V_{C ; R_{\text {cut }}}\right)-\delta_{L \pm}\left(V_{C ; R_{\text {cut }}}^{p t}\right),
$$

and measures the departure from the cut-off point Coulomb scattering due to the full cut-off interaction $V_{N}+V_{C ; R_{\text {cut }}}$. For sufficiently large $R_{\text {cut }}$ this is expected to approach the usual Coulomb-modified nuclear phase shift $\delta_{L \pm}(N)$ introduced in Eq. (4). In terms of the partial wave amplitudes, Eqs. (14) and (16), the $T_{L \pm}^{(\mathrm{IV})}(N)$ can be written

$T_{L \pm}^{(\mathrm{IV})}(N)=\frac{T_{L \pm}\left(V_{N}+V_{C ; R_{\mathrm{cut}}}\right)-T_{L \pm}\left(V_{C ; R_{\mathrm{cut}}}^{p t}\right)}{2 i T_{L \pm}\left(V_{C ; R_{\mathrm{cut}}}^{p t}\right)+1}$ 


\section{NUMERICAL RESULTS}

In order to assess the numerical accuracy and stability of the various approximate methods it is very desirable to be able to perform both configuration and momentum space calculations for the same system. For simplicity, we thus make use of the local proton-target optical potential $^{5}$

$$
\begin{aligned}
& \left\langle\mathbf{k}^{\prime}\left|U_{N}\right| \mathbf{k}\right\rangle=\frac{A-1}{A} \rho(q)\left[t^{c}(\epsilon, q)+\sigma \cdot \hat{n} t^{l s}(\epsilon, q)\right], \\
& q=\left|\mathbf{k}^{\prime}-\mathbf{k}\right|,
\end{aligned}
$$

where we take the $t^{a}(\epsilon, q)(a=c, l s)$ as parametrized nucleon-nucleon transition amplitudes obtained by fitting the empirical on-shell amplitudes ${ }^{7}$ as a function of $N N$ scattering energy $\epsilon$. In the evaluation of Eq. (20), $\epsilon$ is set equal to half the beam energy in the $N N$ center of mass frame. The corresponding configuration space interaction was obtained numerically. The Coulomb interaction was assumed that of a uniformly charged sphere of radius $R_{C}=1.3 A^{1 / 3} \mathrm{fm}$. As a specific example, we apply the methods to $p-{ }^{40} \mathrm{Ca}$ elastic scattering at $200 \mathrm{MeV}$ incident energy.

We first investigate quantitatively the methods (I) and (II) outlined above. These calculations were carried out in configuration space for the assumed local proton $-{ }^{40} \mathrm{Ca}$ interaction discussed above, comprising real and imaginary central and spin-orbit terms. We will show only the calculated proton analyzing power, $A_{y}$, which displays the most sensitivity to the accurate inclusion of the Coulomb interaction. The solid curve in Fig. 1 shows the result of the exact (configuration space) calculations, obtained with the computer program CHUCK $3 .^{8}$ The dot-

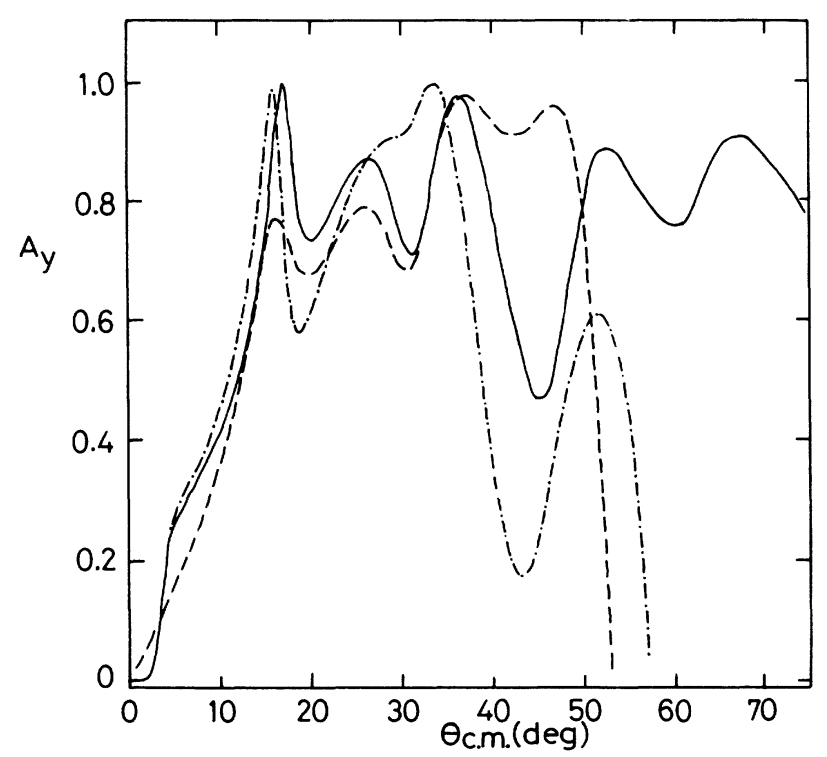

FIG. 1. Calculated vector analyzing powers $A_{y}$ for $p-{ }^{40} \mathrm{Ca}$ elastic scattering at $200 \mathrm{MeV}$. The solid curve includes the Coulomb interaction exactly. The dot-dashed and dashed curves are obtained using methods (I) and (II) of the text, respectively. dashed curve is the result of the nuclear interaction only approach, method (I), while the dashed curve results from the prescription of Picklesimer et al., method (II). The calculations were obtained by substitution of the $T_{L \pm}^{(\mathrm{I})}(N)$ and $T_{L \pm}^{(\mathrm{II})}(N)$ into the expressions of Eqs. (2) and (3). As is clear from the figure, these approximate procedures do not, in any way, reproduce the exact calculations for $A_{y}$ for this system.

In the case of the Vincent-Phatak method we find that, while for low partial waves the treatment is very accurate, the method is numerically inaccurate in the higher partial waves. Similar conclusions were drawn by other authors. ${ }^{2,5}$ Even when the cut-off Coulomb transition amplitudes, $T_{L \pm}\left(V_{N}+V_{C ; R_{\text {cut }}}\right)$, were calculated with high precision in configuration space, the $T_{L \pm}^{(\mathrm{III})}(N)$, obtained using Eq. (12), proved numerically unreliable. Specifically, they showed significant departures from the exact calculations in the $L=20-30$ partial wave region of importance to the $p-{ }^{40} \mathrm{Ca}$ system at $200 \mathrm{MeV}$ considered here. Not surprisingly, this inaccuracy then leads the calculated observables to be sensitive to the particular choice of $R_{\text {cut }}$. As there is no obvious a priori best choice value for $R_{\text {cut }}$, the method is difficult to use with any confidence. We demonstrate a typical set of numerical results in Fig. 2. The figure shows the percentage error in the approximate partial wave transition amplitudes from those of the exact calculation,

$$
\Delta T=\left|T_{L+}^{(i)}(N)-T_{L+}(N)\right| /\left|T_{L+}^{(i)}(N)\right|,
$$

as a function of $L$ for $J=L+\frac{1}{2}$. The circles show the results from the Vincent-Phatak method, Eq. (12), when using a cut-off radius $R_{\text {cut }}=8 \mathrm{fm}$ and, as discussed above, the calculations are in considerable error in the higher

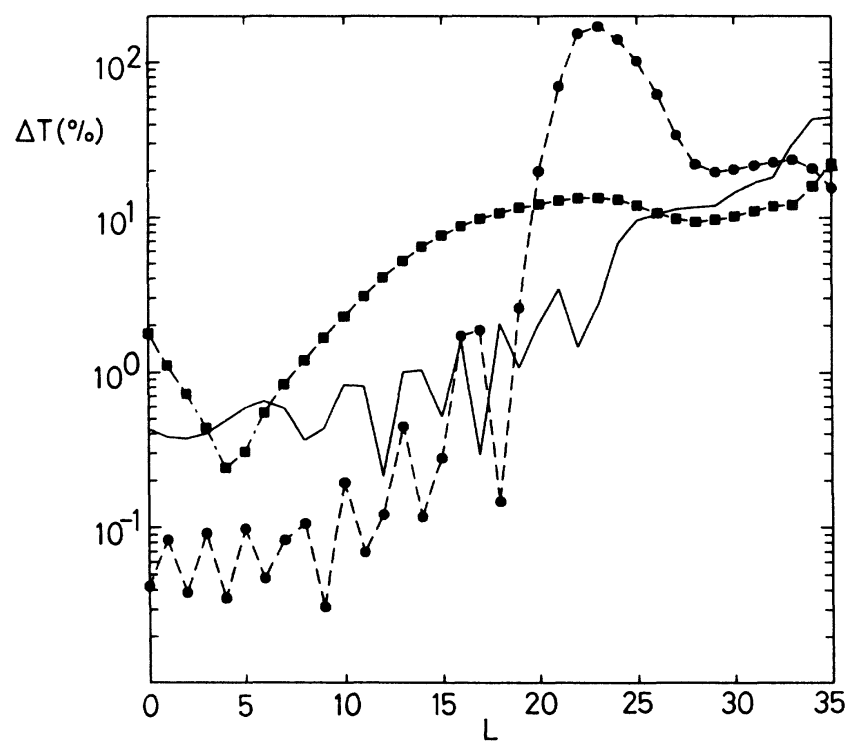

FIG. 2. Percentage errors $\Delta T=\left|T_{L+}^{(i)}(N)-T_{L+}(N)\right| /$ $\left|T_{L+}^{(t)}(N)\right|$ in the $\left(J=L+\frac{1}{2}\right)$ partial wave transition amplitudes calculated using method (II) (squares), method (III) (circles, $R_{\text {cut }}=8 \mathrm{fm}$ ), and method (IV) (solid line, $R_{\text {cut }}=8 \mathrm{fm}$ ). 
partial waves. We present the $R_{\text {cut }}=8 \mathrm{fm}$ calculations as these yielded the best agreement we were able to obtain with the exact $A_{y}$ using the Vincent-Phatak method. This analyzing power (dashed curve) is compared with the exact calculation (solid curve) in Fig. 3. As was discussed in the Introduction, there are significant errors, particularly at the $A_{y}$ minima and at larger scattering angles, where accuracy is required. Also shown in Fig. 2 (squares) are the percentage errors obtained using the approach of Picklesimer et al., leading typically to $10 \%$ errors for partial waves with values $L=15$ onward.

We now consider the proposed approximate method, method (IV), of Eq. (19). The one disadvantage of this method is that it requires two calculations to be performed, namely, a calculation of the nuclear and finite Coulomb problem, $T_{L \pm}\left(V_{N}+V_{C ; R_{\text {cut }}}\right)$, and of the point Coulomb case, $T_{L \pm}\left(V_{C ; R_{c u t}}^{p t}\right)$. The percentage errors in the calculated $T_{L+}^{(\text {IV) }}(N)$, for a cut-off radius $R_{\text {cut }}=8 \mathrm{fm}$, are represented by the solid line in Fig. 2 and the associated $A_{y}$ by the dot-dashed curve in Fig. 3 with significant improvement over the Vincent-Phatak calculation. In this case the agreement with the exact calculation is essentially perfect to scattering angles of order $50^{\circ}$. While the absolute errors in the lower partial waves are greater than those of the Vincent-Phatak approach our method is numerically well behaved across the entire partial wave range. Of course there is no reason why the Vincent-Phatak method should not be implemented in the treatment of the lower partial waves. The rise in the calculated $\Delta T$ at large $L$ simply reflects the fact that the radially cut-off, and consequently $L$ cut-off, partial wave amplitudes entering Eq. (19) eventually fall too rapidly to zero compared with the exact amplitudes.

In Figs. 4 and 5 we demonstrate the systematic im-

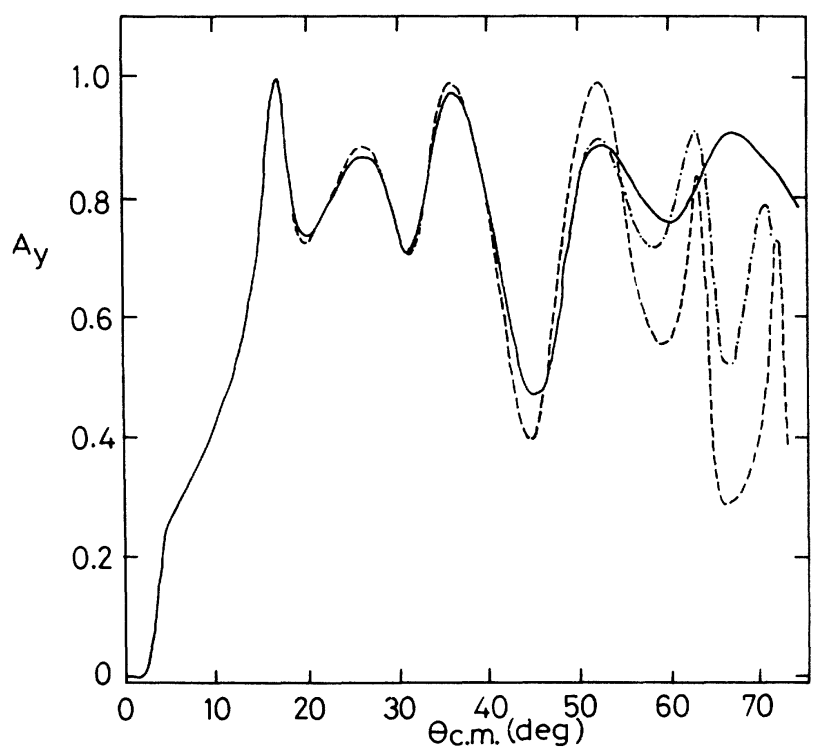

FIG. 3. Calculated vector analyzing powers $A_{y}$ for $p-{ }^{40} \mathrm{Ca}$ elastic scattering at $200 \mathrm{MeV}$. The solid curve includes the Coulomb interaction exactly. The dashed and dot-dashed curves are obtained using methods (III) and (IV) of the text, respectively, with $R_{\text {cut }}=8 \mathrm{fm}$.

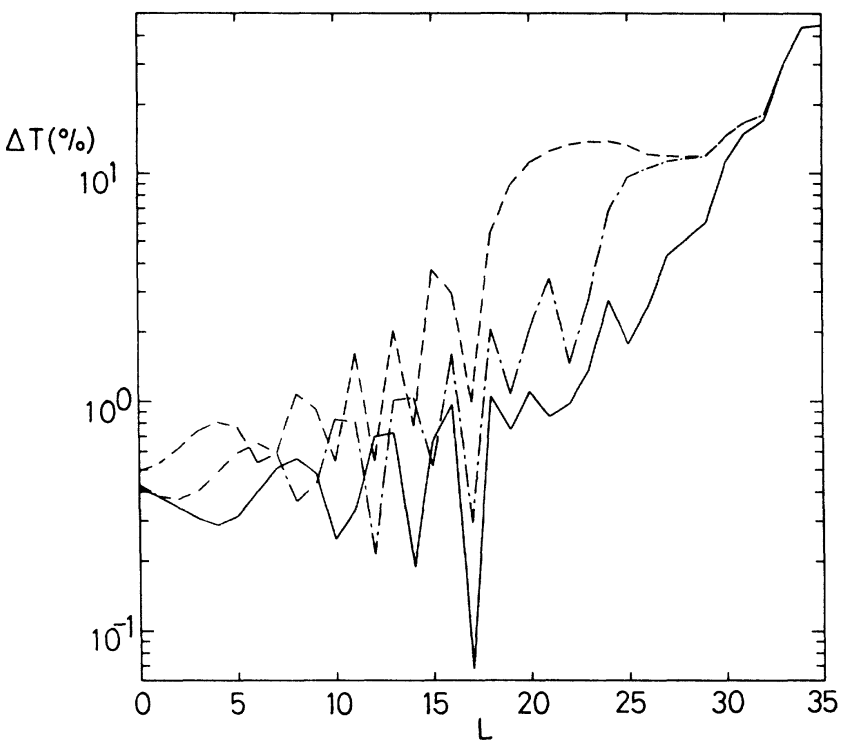

FIG. 4. Percentage errors in the $\left(J=L+\frac{1}{2}\right)$ partial wave transition amplitudes calculated using method (IV). The dashed, dot-dashed, and solid curves show the results obtained with cut-off radii of $R_{\text {cut }}=6,8$, and $10 \mathrm{fm}$, respectively.

provement in the calculated partial wave amplitudes and the convergence toward the exact observables obtained using the method, as a function of cut-off radius used. The dashed, dot-dashed, and solid curves in Fig. 4 show the percentage errors in the $T_{L+}^{(\text {IV })}(N)$ obtained with a cut radius $R_{\text {cut }}=6,8$, and $10 \mathrm{fm}$, respectively. The corresponding analyzing powers are shown by the shortdashed, dot-dashed, and long-dashed curves, respectively, in Fig. 5. In the latter case, $R_{\text {cut }}=10 \mathrm{fm}$, the agreement

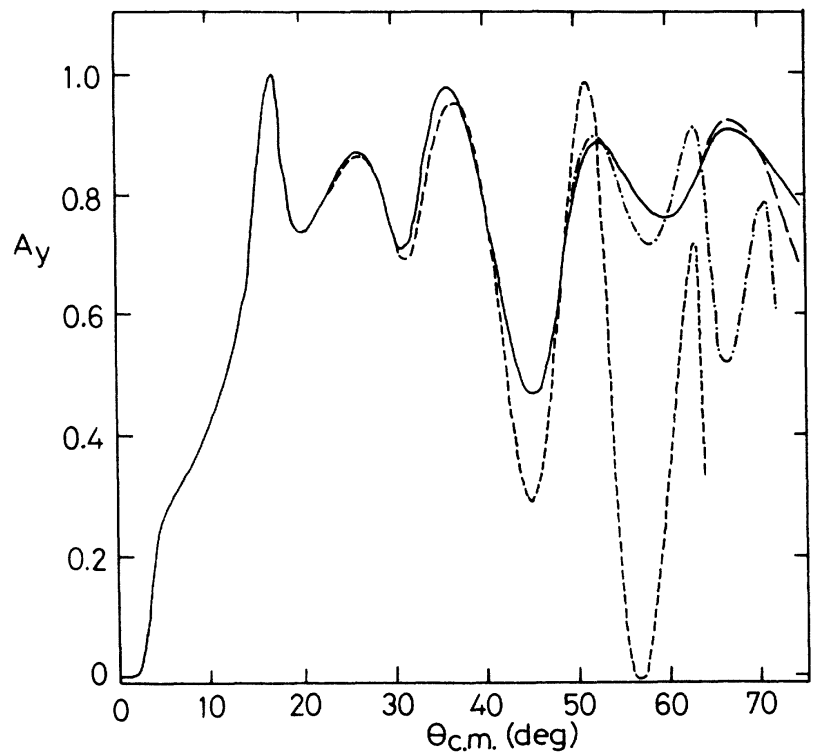

FIG. 5. Calculated vector analyzing powers $A_{y}$ for $p{ }^{40} \mathrm{Ca}$ elastic scattering at $200 \mathrm{MeV}$. The short-dashed, dot-dashed, and long-dashed curves were obtained using method (IV) and a cut-off radius $R_{\text {cut }}=6,8$, and $10 \mathrm{fm}$, respectively. The solid curve includes the Coulomb interaction exactly. 
with the exact result, the solid curve, is excellent up to scattering angles of $70^{\circ}$ in the center of mass. We are able to reproduce these results accurately using both configuration space and momentum space methods. ${ }^{9}$

\section{CONCLUSIONS}

In this paper we presented an approximate procedure for the inclusion of the Coulomb interaction in momentum space calculations of proton elastic scattering. The method is simple to incorporate in momentum space calculations but requires a prior calculation of the cut-off point Coulomb amplitudes, $T_{L \pm}\left(V_{C ; R_{\text {cut }}}^{p t}\right)$, for the system under study. More importantly, the method is well behaved numerically and the accuracy of the partial wave transition amplitudes and the deduced elastic scattering observables is found to improve systematically with increasing radial cut-off, $R_{\text {cut }}$. We have shown that the procedure is capable of producing accurate quantitative calculations of spin-dependent scattering observables for modest values of the Coulomb cut-off radius. The application of the method to full folding calculations of proton-nucleus scattering will be presented elsewhere.

\section{ACKNOWLEDGMENTS}

The financial support of the Instituto Nacional de Investigação Científica and of the British Council (for R.C.) and of the United Kingdom Science and Engineering Research Council (SERC) in the form of Grants GR/F/4105.1 and GR/F/1086.6 (for J.A.T.) is gratefully acknowledged.
*On leave from Physics Department, Instituto Superior Técnico, Lisbon, Portugal.

${ }^{1}$ E. F. Redish and K. Stricker-Bauer, Phys. Rev. C 35, 1183 (1987); H. F. Arellano, F. A. Brieva, and W. G. Love, Phys. Rev. Lett. 63, 605 (1989).

${ }^{2}$ Ch. Elster, T. Cheon, E. F. Redish, and P. C. Tandy, Phys. Rev. C 41, 814 (1990).

${ }^{3}$ A. K. Kerman, H. McManus, and R. M. Thaler, Ann. Phys. (N.Y.) 8, 551 (1959).

${ }^{4}$ Program LpotP, M. J. Paez, M. E. Sagen, and R. H. Landau, Comput. Phys. Commun. 52, 141 (1988).
${ }^{5}$ A. Picklesimer, P. C. Tandy, R. M. Thaler, and D. H. Wolfe, Phys. Rev. C 30, 1861 (1984).

${ }^{6}$ C. M. Vincent and S. C. Phatak, Phys. Rev. C 10, 391 (1974).

${ }^{7}$ R. C. Johnson and D. C. Martin, Nucl. Phys. A192, 496 (1972); A211, 617 (1973).

${ }^{8}$ Program CHUCK3, P. D. Kunz (unpublished); extended version of J. R. Comfort (unpublished).

${ }^{9}$ The momentum space calculations were carried out using an extensively revised version (LPOTPS) of the program LPOTP of Ref. 4. 